\title{
IMPLICAÇÕES DO USO DE TEORIA-K NA ESTIMATIVA DOS MOMENTOS ESTATÍSTICOS DE SEGUNDA ORDEM
}

\author{
Janete G. Lira $^{1, *}$, Leandro L Gonzales ${ }^{1,2}$, Matheus H. S. Ferreira ${ }^{1}$, Felipe D. Costa ${ }^{1}$ \\ ${ }^{1}$ Universidade Federal do Pampa, Campus Alegrete, RS, Brasil \\ ${ }^{2}$ Instituto Federal Sul-rio-grandense, Campus Pelotas, RS, Brasil \\ *janete.glira@gmail.com
}

RESUMO: Neste trabalho, são comparadas duas técnicas para a estimativa dos fluxos turbulentos em um modelo de ordem 1.5, durante o período noturno. Na primeira, utiliza-se a relação fluxo gradiente para estimativa dos fluxos, enquanto que na segunda os fluxos são estimados diretamente a partir da energia cinética turbulenta do escoamento. Os resultados mostram que, quando a relação fluxo gradiente é utilizada, o comportamento complexo do escoamento é reproduzido apenas de forma periódica.

ABSTRACT: In this work, two techniques are compared in order to estimate the turbulent fluxes during the night in a model of 1.5 order. In the first one, it was used the flux gradient relationship to estimate the fluxes, while in the second one the fluxes are estimated directly from the turbulent kinetic energy of the flow. The results showed that, when the flux gradient relationship is used, the complex behavior of the flow is reproduced just in a periodic way.

\section{INTRODUÇÃO}

A descrição do comportamento complexo do escoamento turbulência na camada limite estável (CLE) é uma tarefa muito difícil para modelos numéricos. Um dos principais problemas está na estimativa dos fluxos turbulentos na CLE. Geralmente, esses fluxos são estimados utilizando uma relação fluxo gradiente conhecida como teoria $\mathrm{K}$, na qual os 
coeficientes de difusão turbulenta dependem de alguma função de estabilidade prescrita. Todavia, apesar de representar de forma adequada algumas características médias do escoamento turbulento na CLE, estes modelos não são capazes de reproduzir, por exemplo, o caráter intermitente da turbulência em condições de forte estabilidade.

Neste trabalho, é comparado o desempenho de modelo atmosférico simplificado, utilizando teoria $\mathrm{K}$ no cálculo dos fluxos turbulentos, sem a utilização de funções de estabilidade, com mesmo esquema utilizando o fechamento de turbulência proposto por Costa et al. (2011), que é capaz de reproduzir o comportamento intermitente da turbulência na CLE, e será descrito na próxima seção.

\section{MODELO}

Para uma atmosfera seca, horizontalmente homogênea, livre dos efeitos de advecção onde a divergência do fluxo radiativo é desconsiderada e utilizando o balanço de energia em superfície, as equações que descrevem o escoamento atmosférico na CLE podem ser escritas

$$
\begin{aligned}
& \frac{\partial u}{\partial t}=f\left(v-v_{G}\right)-\frac{\partial\left(\overline{u^{\prime} w^{\prime}}\right),}{\partial z}(1) ; \quad \frac{\partial v}{\partial t}=f\left(u_{G}-u\right)-\frac{\partial\left(\overline{v^{\prime} w^{\prime}}\right)}{\partial z} \quad(2) \quad \frac{\partial \theta}{\partial t}=-\frac{\partial\left(\overline{w^{\prime} \theta^{\prime}}\right)}{\partial z} \\
& \frac{\partial \theta_{g}}{\partial t}=\frac{1}{C_{g}}\left(I_{\downarrow}-\sigma \theta_{g}^{4}-H_{0}\right)-k_{m}\left(\theta_{g}-\theta_{m}\right) \\
& \frac{\partial E}{\partial t}=-\overline{u^{\prime} w^{\prime}} \frac{\partial u}{\partial z}-\overline{u^{\prime} w^{\prime}} \frac{\partial u}{\partial z}+\frac{g}{\theta} \overline{w^{\prime} \theta^{\prime}}-\left[\frac{\partial \overline{E^{\prime} w^{\prime}}}{\partial z}\right]-\varepsilon
\end{aligned}
$$

onde $u$ e $v$ são as componentes do vento, $u_{G}$ e $v_{G}$ são as componentes do vento geostrófico no topo do domínio vertical, $f$ é o parâmetro de Coriolis, $\theta_{g}$ é a temperatura da superfície do solo, $C_{g}$ é a capacidade térmica do solo por unidade de área, $I_{\downarrow}$ representa a radiação de onda longa proveniente da atmosfera, $H_{0}$ é o fluxo de calor sensível na superfície e $\theta_{m}$ é a temperatura do substrato e $\varepsilon=c_{\varepsilon}(\alpha E)^{3 / 2} / l_{m}$ é taxa de dissipação viscosa.

Geralmente, os fluxos turbulentos são parametrizados utilizando teoria $\mathrm{K}$, onde o fluxo de uma determinada quantidade pode ser escrito como: $-\overline{\chi^{\prime} w^{\prime}}=K_{i} \partial \chi / \partial z$. Onde $K_{i}$ são os coeficientes de difusão: $K_{m}, K_{h}=K_{m}$ e $K_{E}=K_{m} / 2.5$. $K_{m}$ é parametrizado como $K_{m}=\sqrt{\alpha E} l_{m}$, 
onde $\alpha=1 / 5,5$ e $l_{m}$ é o comprimento de mistura, neste trabalho assumido como sendo o do caso neutro.

Costa et al. (2011) propuseram, além da não utilização de funções de estabilidade, um fechamento de turbulência onde os fluxos dependem diretamente da intensidade turbulenta

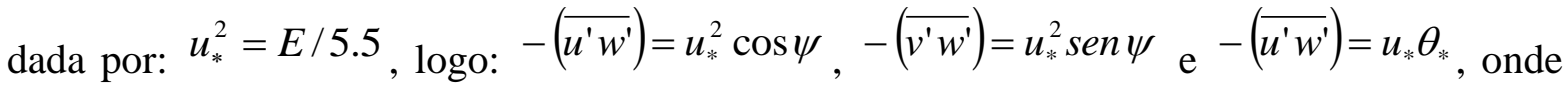
$\theta_{*}$ é a escala de temperatura. É importante destacar que apenas nos cálculos da escala de temperatura e do fluxo turbulento de ECT é utilizada teoria K. Com estas mudanças a equação (5), pode ser reescrita como:

$$
\frac{\partial E}{\partial t}=S u_{*}^{2}-R i S u_{*}^{2}+\left[K_{E} \frac{\partial E}{\partial Z}\right]-c_{\varepsilon} \frac{(\alpha E)^{3 / 2}}{l_{m}}
$$

Finalmente, todas as equações foram discretizadas em um domínio vertical de 50 m, o qual é dividido em $n$ níveis entre o solo e seu topo e integradas, pela utilização do método RungeKutta de quarta ordem.

\section{RESULTADOS}

A figura 1 mostra que quando utilizado apenas 1 nível atmosférico (figura 1a e 1b), ambos os esquemas utilizados apresentam apenas oscilações periódicas no estado desconectado. Todavia, quando são considerados mais níveis entre a superfície e o topo do domínio, a complexidade das soluções para forçantes mecânicos intermediários aumenta, desde que não se utilize teoria K (figuras 1c e 1d). Mesmo com a utilização de 15 níveis atmosféricos, o esquema utilizando teoria $\mathrm{K}$ leva a soluções periódicas, enquanto que o esquema similar leva a soluções que correspondem, qualitativamente, ao comportamento imprevisível do escoamento atmosférico em condições muito estáveis.

Outro ponto importante a ser destacado é que ambos os esquemas reproduzem o acoplamento atmosférico. Entretanto, a mistura turbulenta, quando utilizada teoria $\mathrm{K}$ muito intensa (figuras 1e e 1f), isto se deve ao aumento dos fluxos turbulentos após a conexão, este mesmo aumento não é verificado no esquema sem teoria $\mathrm{K}$ porque o termo de destruição térmica é diretamente proporcional a intensidade de $E$, atuando desta forma como um sumidouro mais eficiente de ECT. 

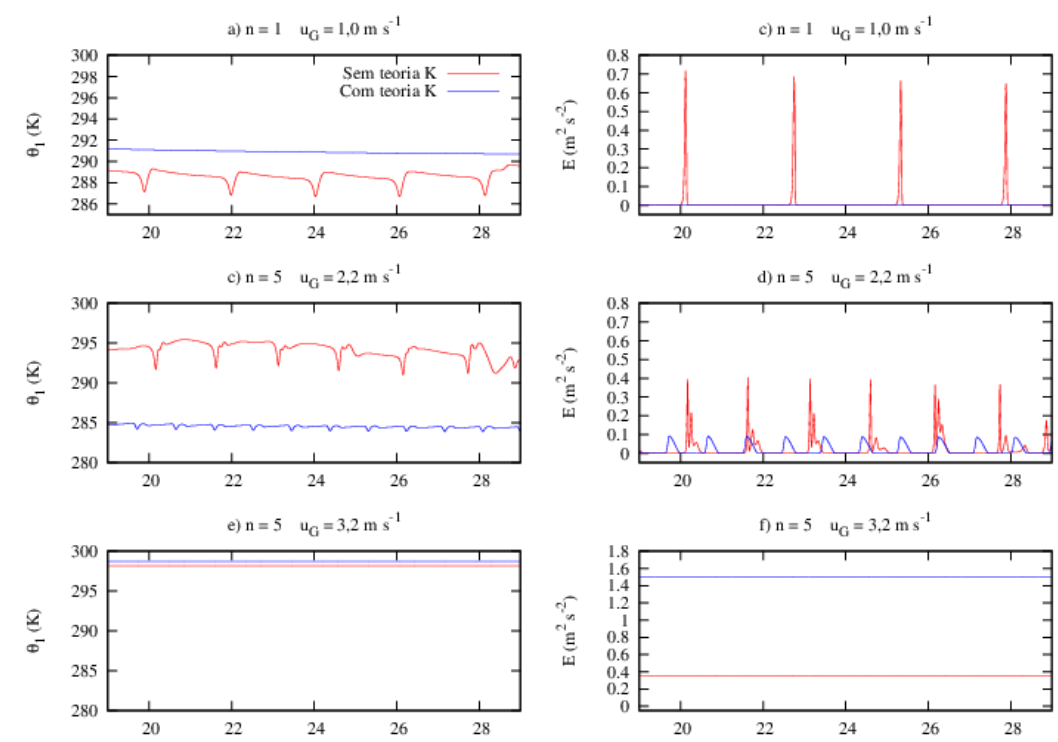

Figura 1: Séries temporais de temperatura e ECT a 5 m, para os dois fechamentos de turbulência, indicados pela legenda.

\section{CONCLUSÃO}

Os resultados obtidos a partir da comparação indicam que a utilização de teoria $\mathrm{K}$ para estimar os momentos estatísticos de segunda ordem, pode reduzir o grau de complexidade das soluções. Entretanto, este resultado carece de uma análise matemática aprofundada do ponto de vista dinâmico do sistema para sustentar esta hipótese.

\section{REFERÊNCIAS}

COSTA, F. D.; ACEVEDO, O. C.; MOMBACH, J. M. C.; DEGRAZIA, G. A. A simplified model for intermittent turbulence in the nocturnal boundary layer. J. Atmos. Sci., v. 68, n. 8, p. 1714-1729, 2011. 